\title{
Neorchesella, New Genus of Springtails from Mexico (Collembola: Entomobryidae: Orchesellinae $)^{1}$
}

\author{
José A. Mari Mutt ${ }^{2}$ \\ ABSTRACT \\ Neorchesella mexicana, new genus and species from Tamaulipas State, \\ Mexico is described and differentiated from its closest relatives. A revised \\ key to the genera of Orchesellinae with 6-segmented antennae is included.
}

\section{INTRODUCTION}

This paper describes the second endemic Mexican genus of the subfamily Orchesellinae, the first being Dicranorchesella Mari Mutt. ${ }^{3}$ The new taxon is clearly related to Orchesella Templeton and may well have originated from an ancient member of that genus. The name Neorchesella emphasizes this similarity.

\section{RESULTS}

\section{NEORCHESELLA MARI MUTT, NEW GENUS}

Orcheselline in general appearance (plate I, fig. 1). Fourth abdominal segment not over 1.5 times length of third segment. Scales absent. Body covered by numerous ciliated macro- and microchaetae. Inner margin of tibiotarsi with rows of smooth (finely striated) setae. Antennae 6-segmented, apical segment (Ant. 6) and anterior third of Ant. 5 not clearly annulated but with setae arranged in definite whorls. Division between Ants. 3 and 4 distinct. Labral chaetotaxy follows formula 5, 5, 4; all setae smooth. Labral papillae (plate II, fig. 6) apically pointed, similar to that of Orchesella species. Eyes $2+2$. Postantennal organ absent. General head and body macrochaetotaxy as in figures 4 and 8 , definitely polychaetotic. Claw structure (plate III, fig. 11) as in most Orchesellinae; unguis with 2 paired inner teeth and a distal unpaired tooth. Unguiculus with an outer tooth. Pretarsal setae present. Tenent hair lanceolate. Manubrial organ not seen as sex of specimens not determined. Dental

${ }^{1}$ Manuscript submitted to Editorial Board October 31, 1979.

${ }^{2}$ Department of Biology, University of Puerto Rico, Mayagüez, P. R. The author wishes to express his gratitude to Dr. Kenneth A. Christiansen, Department of Biology, Grinnell College, Iowa, for his gift of the specimens. He also thanks the staff of the Faunistics Section of the Illinois Natural History survey for providing space and equipment during a recent stay there. Much of the present work was completed then.

${ }^{3}$ Mari Mutt, J. A., 1977. Dicranorchesella, a new genus of springtails from Mexico (Collembola: Entomobryidae). Proc. Entomol. Soc. Wash., 79 (3): 377-382. 


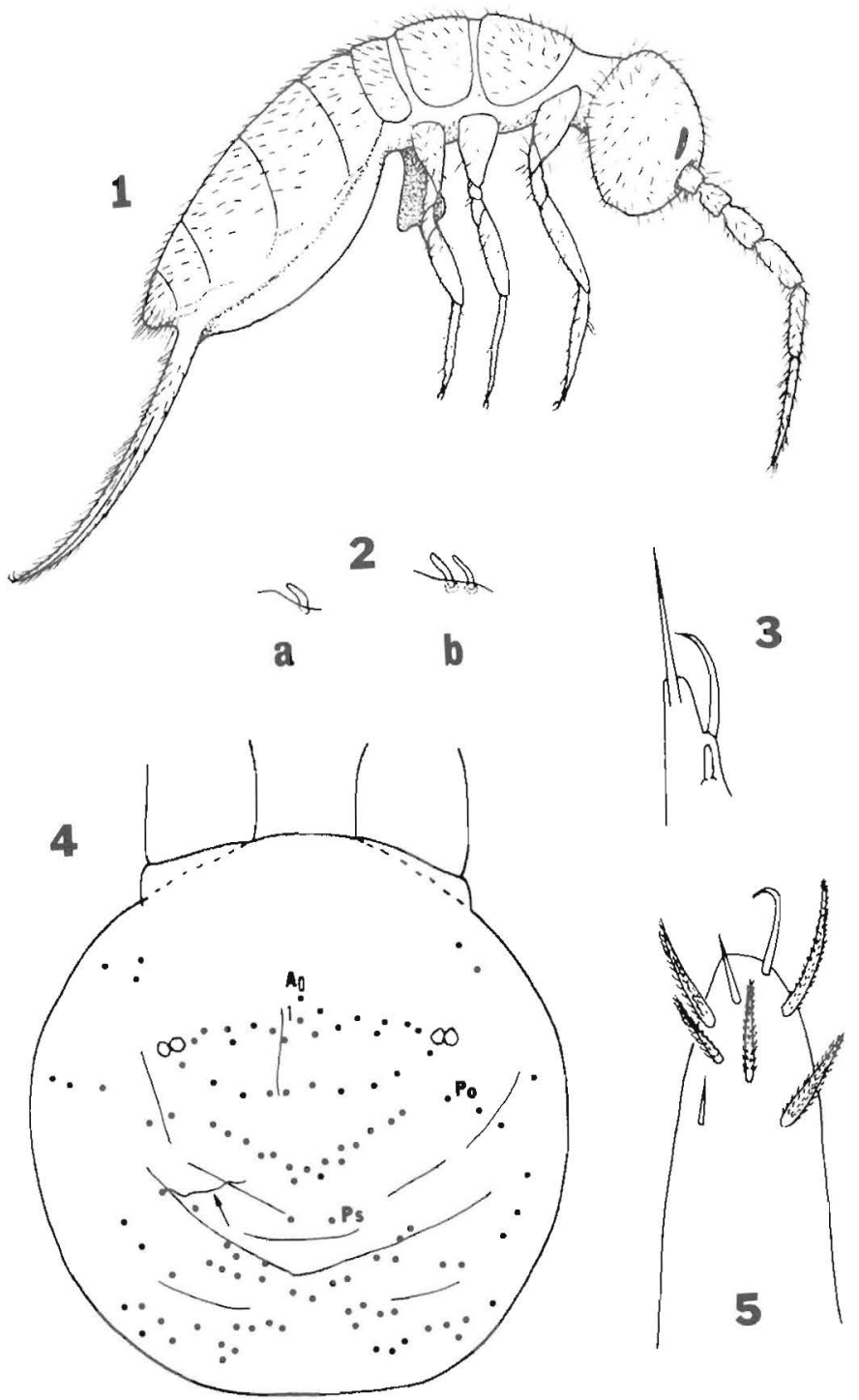

Plate I. Fig. 1.-Habitus. Fig. 2.-a-sensillum on apex of Ant. 2, b-sensilla on apex of Ant. 3. Fig 3.-Outer labial papilla and its differentiated rodlike seta. Fig. 4.-Head macrochaetotaxy, each dot represents a seta. All lines (except the one indicated by an arrow which stands for a lasiotrichium) represent infoldings of the cuticle caused by mounting procedure; some setae may be hidden in these depressions. Nomenclature is explained by Mari Mutt $\left({ }^{4}\right)$. Fig. 5.-Apex of Ant. 6 showing position of smooth hooked seta.

${ }^{4}$ Mari Mutt, J. A., 1979. A revision of the genus Dicranocentrus Schott (Insecta; Collembola: Entomobryidae). Agr. Exp. Stn. Univ. P. R. Bull. 259. 

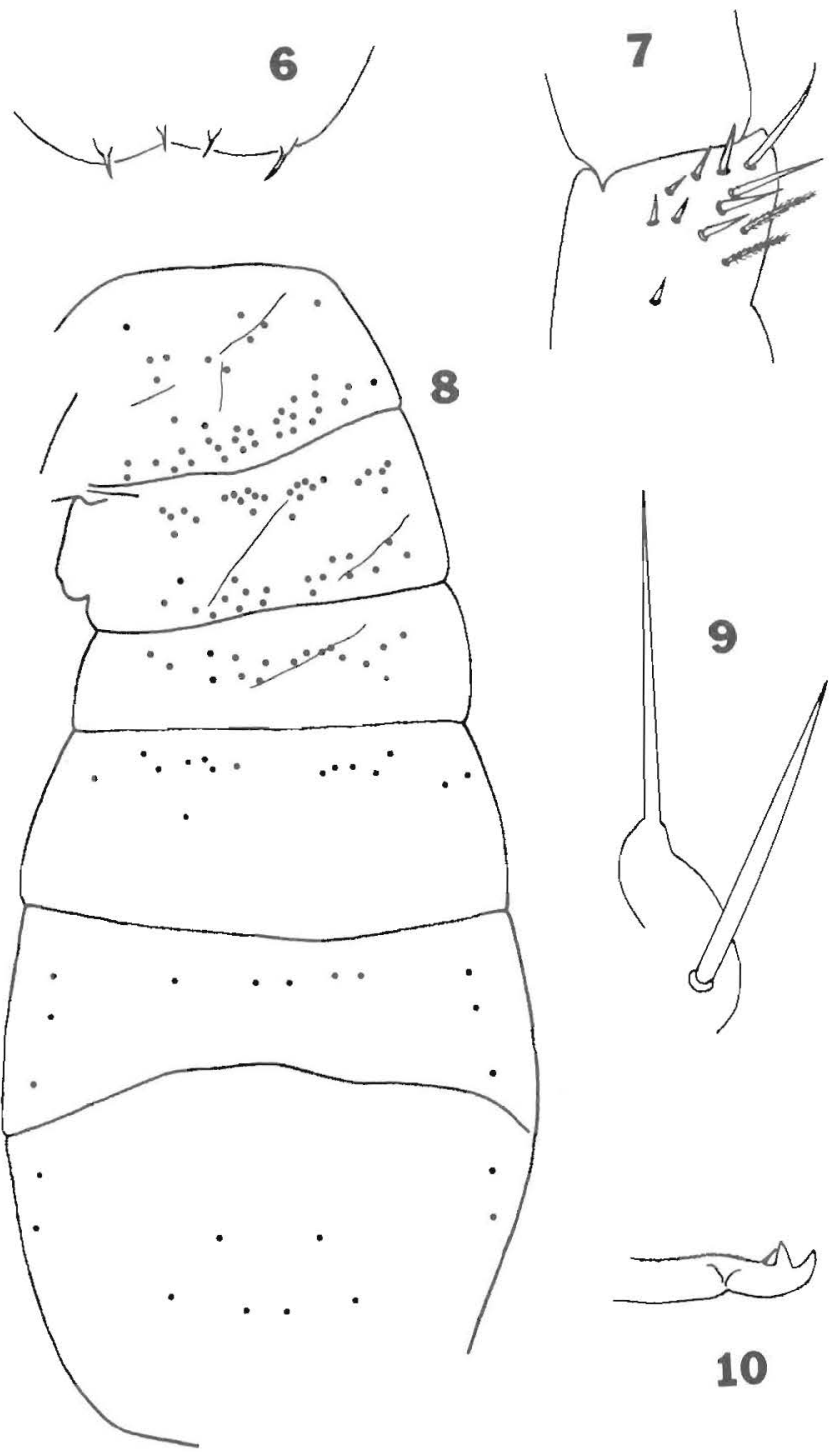

P'LATE II. Frg.6.-Labral papillae. Fig, 7.-Trochanteral organ. Fig. 8.-Body macrochaetotixy; each dot represents seta, lines stand for infoldings of the cuticle due to mounting procedure. Figs. 9.-Labial palp. Figs. 10.-Mucro. 


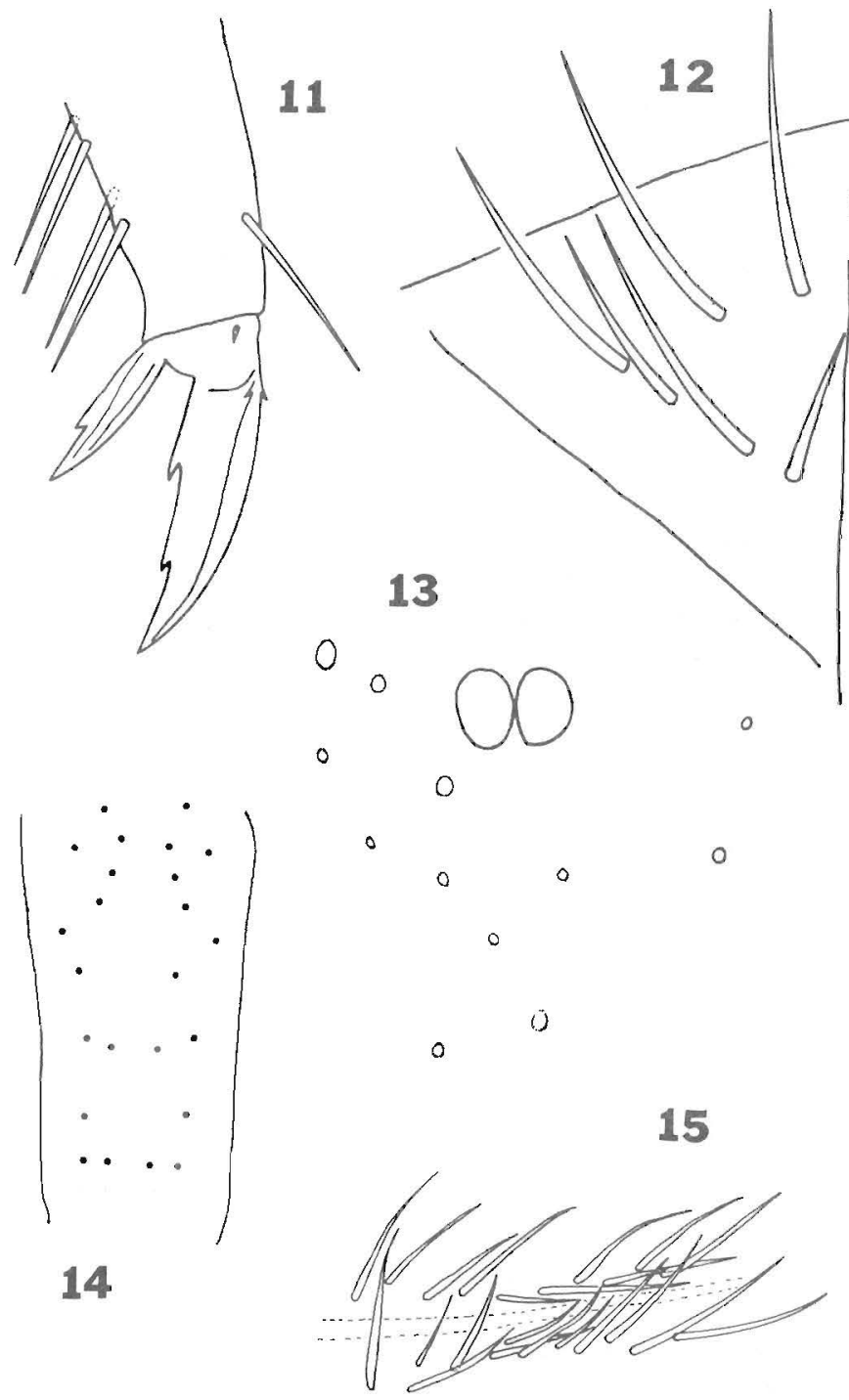

PLATE III. FIG. 11.-Structure of metathoracic claw. Fig. 12.-Chaetotaxy of labial triangle. Fig. 13.-Chaetotaxy of the area adjacent to the eyes, each circle represents a seta. Fig. 14.-Distribution of smooth erect setae on dorsum of manubrium, bottom of figure leads to dens. Fig. 15.-Arrangement of setae along cephalic groove, immediately behind labial triangle. The right side of the figure points to the triangle. 
spines absent. Mucro (plate II, fig. 10) typical of subfamily, with 2 teeth and a basal spine.

Type Species

Neorchesella mexicana n. sp.

Diagnosis

Neorchesella is closely related to Orchesella Templeton and Dicranorchesella Mari Mutt. These genera may be readily separated by means of the following key:

KEY TO THE GENERA OF ORCHESELLINAE POSSESSING 6-SEGMENTED ANTENNAE

1. Scales absent . . . . . . . . . . . . . . . . . . . . . . . 2

Scales present ................................ 3

2. Eyes $8+8$ on dark patches; tibiotarsi without smooth setae (with exception of opposite seta to tenent hair of metathoracic legs, which is present throughout the subfamily); tenent hair apically knobbed; apex of Ant. 6 with pin seta ............ Orchesella Templeton Eyes $2+2$ on very light orange patches; inner margin of tibiotarsi with rows of smooth setae; tenent hair apically pointed; apex of Ant. 6 without pin seta

Neorchesella NEW GENUS

3. Scales variable in size and shape but not fusiform, usually apically rounded or truncated; scales present on first 4 antennal segments and throughout legs; microchaetae scarce on head and body, restricted mainly to region between antennal bases... Dicranocentrus Schött Scales similar in size and fusiform, absent from antennae and legs; microchaetae abundant on head and body

Dicranorchesella Mari Mutt

Neorchesella mexicana MARI MUTT, NEW SPECIES

Length up to $2.0 \mathrm{~mm}$. Antennae half as long as head and body combined, apical segment (Ant. 6) 1.4 times longer than Ant. 5. Apex of Ant. 6 without pin seta but with a long, apically hooked seta (plate I, fig. 3). Ant. 5 sense organ as in figure $2 \mathrm{~b}$ plate I. Apex of Ant. 2 with a single sensilla (plate I, fig. 2a) homologous to those on Ant. 5. Labral papillae as in figure 6 plate II. Chaetotaxy of labial triangle as in figure 12 plate III, all setae smooth. Setae of maxillary palp as in figure 9 plate II. Differentiated seta of outer labial papilla (plate I, fig. 3) short, placed far back on its papilla. Setae along ventral cephalic groove as in figure 15, plate III, and all smooth. Great majority of other ventral head setae also smooth. Eyes (plates I, III, figs. 4 , 13) $2+2$ surrounded by very light orange pigment. Head macrochaetotaxy as in figure 4, setae $A_{I}$ and Ps 
present. Trochanteral organ (plate II, fig. 7) of 10 spine-like setae. Inner margin of tibiotarsi with at least a double row of stout, smooth (finely striated) setae (e.g. plate III, fig. 11). A smooth seta present opposite tenent hair of metathoracic legs. Claw structure as in figure 11 plate III. Unguis with a pair of small basal outer teeth, a pair of large basal inner teeth, and a distal unpaired tooth. Unguiculus with a fairly large tooth on one outer lamella. Tenent hair apically pointed. Body macrochaetotaxy as in figure 8 plate II. Dorsum of manubrium with erect smooth setae arranged as in figure 14 plate III. Dental spines absent. Mucro (plate II, fig. 10) with two teeth and a basal spine.

Material Examined and Repository:

Mexico, Tamaulipas State, Rancho del Cielo, $8 \mathrm{~km}$ north of Gómez Farías, March 9, 1969, J. Reddell, col. Holotype and 4 paratypes (3 are immatures).

The holotype and 3 paratypes are in the Illinoiis Natural History Survey; the remaining specimen is in the author's collection.

\section{RESUMEN}

Se describen el nuevo género y la nueva especie Neorchesella mexicana, del Estado de Tamaulipas, México, y se diferencia de los taxones más semejantes. Se presenta una clave revisada para los géneros de Orchesellinae cuyos miembros poseen antenas de seis artejos. 